\title{
Blending the distinctions among groups of minor bodies: a portrait of the Centaur-comet "transition" object P/2010 C1 (Scotti) ${ }^{\star}$
}

\author{
E. Mazzotta Epifani ${ }^{1}$, D. Perna ${ }^{2}$, J. Licandro ${ }^{3,4}$, M. Dall'Ora ${ }^{1}$, P. Palumbo ${ }^{5}$, E. Dotto ${ }^{6}$, M. A. Barucci ${ }^{2}$, J. R. Brucato ${ }^{7}$, \\ V. Della Corte ${ }^{8}$, and G. P. Tozzi ${ }^{7}$
}

\author{
1 INAF - Osservatorio Astronomico di Capodimonte, via Moiariello 16, 80131 Napoli, Italy \\ e-mail: epifani@oacn.inaf.it \\ 2 LESIA - Observatoire de Paris, CNRS, UPMC, Université Paris-Diderot, 5 place Jules Janssen, 92195 Meudon Cedex, France \\ 3 Instituto de Astrofísica de Canarias, c/vía Láctea s/n, 38200 La Laguna, Tenerife, Spain \\ ${ }^{4}$ Departamento de Astrofísica, Universidad de La Laguna, 38205 La Laguna, Tenerife, Spain \\ 5 Universitá Parthenope, Dip. Scienze Applicate, Centro Direzionale Isola C4, 80143 Napoli, Italy \\ ${ }^{6}$ INAF - Osservatorio Astronomico di Roma, via Di Frascati 33, 00040 Monte Porzio Catone, Roma, Italy \\ 7 INAF - Osservatorio Astrofisico di Arcetri, Largo Enrico Fermi 5, 50125 Firenze, Italy \\ 8 INAF - IAPS, via Del Fosso del Cavaliere 100, 00133 Roma, Italy
}

Received 27 September 2013 / Accepted 18 March 2014

\section{ABSTRACT}

\begin{abstract}
Aims. We present a portrait of the active Centaur P/2010 C1 (Scotti), observed with a well developed comet-like activity (central diffuse coma condensation and an extended sharp tail-like structure) at the heliocentric distance of $r_{\mathrm{h}}=5.5 \mathrm{AU}$.

Methods. We analyse multicolour $(B, V, R$, and $I)$ images taken at the TNG telescope to characterise the dust coma of the active Centaur and to investigate its photometry, colours, and dust production, and to gain hints to its nucleus size.

Results. $\mathrm{P} / 2010 \mathrm{C} 1$ (Scotti) has a small nucleus $(<4.8 \mathrm{~km}$ in radius), with visual colour $V-R=0.49 \pm 0.03$ typical of the nuclei of Jupiter-family comets, but with a dust coma much redder $(B-I=2.37 \pm 0.13)$ than both the Sun and the typical comet mucleus. It has an integrated $R$ magnitude of $m_{R}=21.41 \pm 0.02$ and $R$-A $f \rho=34 \pm 2 \mathrm{~cm}$ (all measured in the reference aperture of radius $\left.\phi=2.0^{\prime \prime}\right)$, which is much lower than has been measured for other active Centaurs at similar heliocentric distances. Its dust production rate, $Q_{\mathrm{d}}=0.1-15 \mathrm{~kg} / \mathrm{s}$, depicts $\mathrm{P} / 2010 \mathrm{C} 1$ (Scotti) as a rather weak dust emitter, with a dust-loss rate comparable to that of the weakest Jupiter-family comets at shorter heliocentric distance and to the weakest active Centaur characterised to now.
\end{abstract}

Key words. comets: general - comets: individual: P/2010 C1 (Scotti)

\section{Introduction}

Several minor bodies reside in the region of the solar system between Jupiter's and Neptune's orbits, moving on highly chaotic and dynamically unstable orbits. These bodies are often called Centaurs, but, surprisingly, there is no uniformly accepted definition of these bodies. Following Jewitt (2009), we define a Centaur as a body whose orbit meets the following conditions: 1) $a_{\mathrm{J}}<q, a<a_{\mathrm{N}}$, i.e., its perihelion distance $q$ and semimajor axis $a$ lie within the semimajor axis of Jupiter $\left(a_{\mathrm{J}}=5.2 \mathrm{AU}\right)$ and Neptune $\left(a_{\mathrm{N}}=30 \mathrm{AU}\right)$; and 2$)$ its orbit is not in 1:1 mean-motion resonance with any planet.

With orbital lifetimes less than $10^{7}$ years (Holman \& Wisdom 1993), the Centaurs are brief residents in the region between the gas giant planets, and those who survive the dynamical environment in this region may become Jupiter family comets (Levison \& Duncan 1997). They are therefore considered "transition" objects from the group of inactive Kuiper Belt objects to the one of active Jupiter family comets, i.e. from the

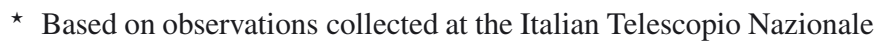
Galileo (TNG), operated on the island of La Palma by the Centro Galileo Galilei of the INAF (Istituto Nazionale di Astrofisica) at the Spanish Observatorio del Roque de los Muchachos of the Instituto de Astrofísica de Canarias. cold, far Kuiper Belt region to the inner solar system. Most recent results (Bauer 2013) point in fact to a cometary origin for many objects in the group, meaning that a Centaur is made of the same material as a comet, may have been a comet in the past, and/or may become a comet in the future. Actually, a few Centaurs ${ }^{1}$ ( 22 objects in a family of $\sim 150$ members, i.e., around $15 \%$ of the sample, as of September 2013) have been observed with a diffuse dust coma and/or a tail in optical images.

For the purposes of the present paper, the dynamical behaviour of the active Centaurs (plus other small bodies with similar characteristics, as detailed below) in the past 300 years have been derived by means of the Horizons On-Line Ephemeris System by JPL (Giorgini et al. 1996), which provides access to key solar system data and to the dynamic production of highly accurate ephemerides. The system is an integrator that takes the perturbing forces (gravitational attraction) of the major solar system bodies into account, while integrating the orbital elements of the target body backward and forward. For small solar system bodies, the system can compute also a close-approach table, in which a "nominal" giant planet encounter is flagged (where "nominal" means "highest probability for the given orbit

\footnotetext{
See www.physics.ucf.edu/ yfernandez/cometlist.html for a continuously updated list.
} 
Table 1. Active Centaurs and transitional comets (as per November 2013).

\begin{tabular}{cccccccc}
\hline \hline Group $^{a}$ & & $q^{b}[\mathrm{AU}]$ & $a^{c}[\mathrm{AU}]$ & $e^{d}$ & $i^{e}\left[{ }^{o}\right]$ & $\mathrm{P}^{f}[\mathrm{yr}]$ & Reference \\
\hline $\mathrm{A}$ & 29P/S-W 1 & 5.724 & 5.987 & 0.044 & 9.4 & 14.7 & $(1)$ \\
& 39P/Oterma & 5.471 & 7.251 & 0.245 & 1.9 & 19.5 & $(2)$ \\
95P/Chiron & 8.454 & 13.710 & 0.383 & 6.9 & 50.7 & $(3)$ \\
& 165P/LINEAR & 6.830 & 18.027 & 0.621 & 15.9 & 76.4 & $(4)$ \\
166P/NEAT & 8.564 & 13.873 & 0.384 & 15.4 & 51.9 & $(5)$ \\
167P/CINEOS & 11.788 & 16.167 & 0.269 & 19.1 & 64.8 & $(6)$ \\
174P/Echeclus & 5.826 & 10.764 & 0.457 & 4.3 & 35.1 & $(7)$ \\
& C/2001 M10 (NEAT) & 5.303 & 26.662 & 0.801 & 28.1 & 138 & $(8)$ \\
P/2005 S2 (Skiff) & 6.398 & 7.964 & 0.197 & 3.1 & 22.5 & $(9)$ \\
& P/2007 S2 (Lemmon) & 5.549 & 12.551 & 0.555 & 16.9 & 44.1 & $(10)$ \\
& 2003 QD112 & 7.935 & 19.084 & 0.583 & 14.5 & 83.4 & $(11)$ \\
& C/2001 P2 (PanSTARRS) & 6.147 & 9.767 & 0.372 & 9.0 & 30.6 & $(12)$ \\
& P/2010 TO20 (LINEAR-Grauer) & 5.12 & 5.60 & 0.088 & 2.64 & 13.3 & $(13)$ \\
P/2011 S1 (Gibbs) & 7.025 & 8.449 & 0.168 & 2.7 & 24.6 & $(14)$ \\
& C/2012 Q1 (Kowalski) & 9.482 & 26.154 & 0.638 & 45.2 & 134 & $(15)$ \\
C/2013 C2 (Tenagra) & 9.131 & 15.993 & 0.429 & 21.3 & 64 & $(16)$ \\
\hline $\mathrm{B}$ & $\mathrm{P} / 2004$ A1 (LONEOS) & 5.463 & 7.897 & 0.308 & 10.6 & 22.2 & $(17)$ \\
& P/2005 T3 (Read) & 6.202 & 7.507 & 0.174 & 6.3 & 20.6 & $(18)$ \\
& P/2008 CL94 (Lemmon) & 5.436 & 6.170 & 0.121 & 8.3 & 14.4 & $(19)$ \\
P/2010 C1 (Scotti) & 5.235 & 7.065 & 0.259 & 9.1 & 18.8 & $(20)$, this work \\
& P/2010 H5 (Scotti) & 6.026 & 7.144 & 0.157 & 14.1 & 19.1 & $(21)$ \\
P/2011 C2 (Gibbs) & 5.384 & 7.371 & 0.270 & 10.9 & 20 & $(22)$ \\
\hline C & P/1997 T3 (Lagerkvist-Carsenty) & 4.241 & 6.682 & 0.365 & 4.8 & 17.3 & $(23)$ \\
& P/1998 U3 (Jager) & 2.134 & 6.067 & 0.648 & 19.1 & 14.9 & $(24)$ \\
P/2003 L1 (Scotti) & 5.010 & 6.698 & 0.252 & 9.0 & 17.3 & $(25)$ \\
& P/2008 T1 (Boattini) & 3.043 & 4.233 & 0.281 & 2.1 & 8.7 & $(26)$ \\
& P/2010 H4 (Scotti) & 4.821 & 6.624 & 0.272 & 2.3 & 17.1 & $(27)$ \\
\hline
\end{tabular}

Notes. Orbital parameters are from the JPL Horizon Data Base. ${ }^{(a)}$ See text. ${ }^{(b)}$ Perihelion distance. ${ }^{(c)}$ Semi-major axis. ${ }^{(d)}$ Orbital eccentricity. ${ }^{(e)}$ Orbital inclination. ${ }^{(f)}$ Orbital period. ${ }^{(e)}$ Included for its orbital similarity with cometary Centaur 29P.

References. Code for the references (first discovery or discussion on activity): (1) Jewitt (1990); (2) Fernandez et al. (2001); (3) Kowal et al. (1977); (4) Kusnirak \& Balam (2000); (5) Pravdo et al. (2001); (6) Romanishin \& Tegler (2005); (7) Choi et al. (2006); (8) Lawrence et al. (2001); (9) Green (2005); (10) Buzzi et al. (2007); (11) Jewitt (2009); (12) Primak et al. (2011); (13) Lacerda (2013); (14) Gibbs et al. (2011b); (15) Miller et al. (2012); (16) Bressi et al. (2013); (17) Green (2004); (18) Read \& Scotti (2005); (19) Scotti et al. (2009); (20) Scotti (2010); (21) Scotti et al. (2010); (22) Gibbs et al. (2011a); (23) Lagerkvist et al. (2000); (24) Green (1998); (25) Meyer (2003); (26) Boattini et al. (2008); (27) Green (2010).

solution"). The JPL Horizon integrator has been therefore used to investigate possible recent modifications of orbital parameters and therefore to define the three groups (A, B, and C) listed in Table 1.

Group A lists the active Centaurs that have shown a Centaurlike orbit in the past 300 years, which did not experience close encounters with any giant planet or did so, but this had no major effect on their orbital parameters, because their heliocentric distance remained quite chaotic but always within the Centaur zone. Group B lists the active Centaurs that experienced one or more close encounters with Saturn in the past 300 years, which injected them into a more internal than before, still Centaur-like orbit inside the solar system. Group C lists objects presently classified as short-period comets, under transition towards the inner solar system, which experienced one or more close encounters with Saturn that recently perturbed their Centaur-like orbit and turned them into "comets" (see below for more details on this "new group").

The prototype of active Centaur is (2060) Chiron (Kowal et al. 1979; Meech \& Belton 1989; Luu \& Jewitt 1990), which orbits at about 10 AU from the Sun and shows a sustained, though variable, comet-like activity along its orbit. The activity among Centaurs is part of a wider debate about the activity of minor bodies at great distance from the Sun, outside the socalled "water zone". Beyond 5-6 AU, comet-like activity can no longer be explained by classical water ice direct sublimation (Meech \& Svoren 2004). Models of cometary nuclei (e.g., Prialnik 1997; Capria et al. 2000a,b), and laboratory experiments (e.g., Notesco et al. 2003) show that amorphous water ice is highly porous and can contain a large number of (hyper)volatile molecules trapped at time of condensation. The trapped gas could be released upon ice crystallisation, giving rise to dust loss at temperatures too low (and hence at heliocentric distances too large) for crystalline water ice to sublimate; for example, the activity in Chiron has been modelled as a product of crystallisation heating by Prialnik et al. (1995). Many active Centaurs have dust loss rates comparable to or even higher than several active comets at much shorter heliocentric distance: for example, $\mathrm{P} / 2004$ A1 (LONEOS) has a dust production rate around 100-200 $\mathrm{kg} \mathrm{s}^{-1}$ up to $6.5 \mathrm{AU}$ (Mazzotta Epifani et al. 2006, 2011).

The existence of another group of "transition" active minor bodies has been recently postulated (Hahn \& Lagerkvist 2010). These are presently short-period comets, whose secular orbital history is characterised by a more or less recent close encounter with Saturn that modified their previous Centaurlike orbit and turned it into a short-period comet-like one (see Table 1, group C); for example, the presently defined Jupiter family comet P/2008 T1 (Boattini) (Boattini et al. 2008) was (dynamically) a presumably active Centaur up to 2003, when it 
E. Mazzotta Epifani et al.: The active Centaur P/2010 C1 (Scotti)

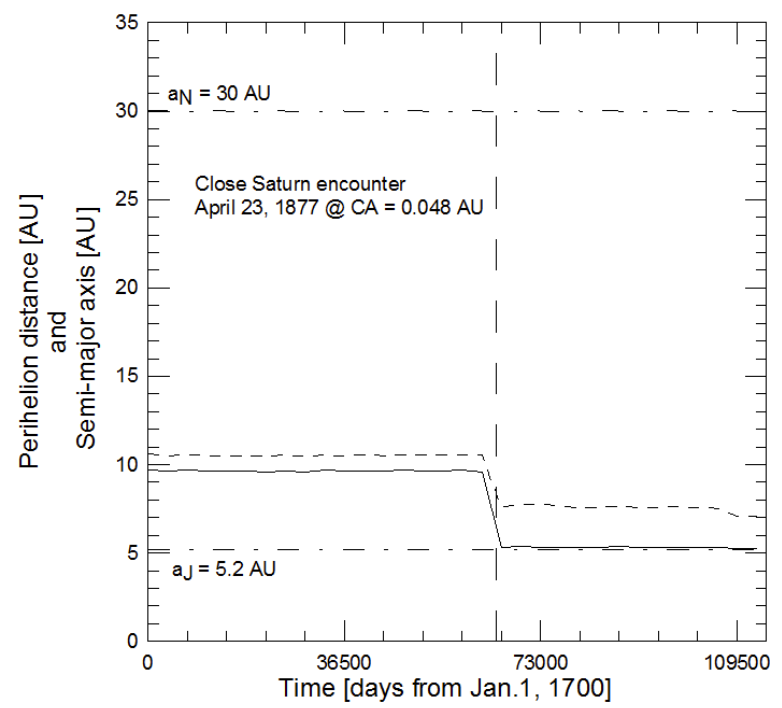

Fig. 1. Long-term dynamical behaviour of active Centaur P/2010 C1 (Scotti). The continuous curve is the perihelion distance, the dotted curve is the semi-major axis, both drastically reduced after a close $(\mathrm{CA}=0.048 \mathrm{AU})$ encounter with planet Saturn, occurred on April 23, 1877 (vertical long-dashed line). The horizontal dot-dashed lines mark the Jupiter $\left(\mathrm{a}_{\mathrm{J}}=5.2 \mathrm{AU}\right)$ and Neptune $\left(\mathrm{a}_{\mathrm{N}}=30 \mathrm{AU}\right)$ semi-major axis, which, following Jewitt (2009), limits the dynamical region of Centaurs.

"turned" into its present class after close encounters with Saturn (and Jupiter). In some cases, they presently belong to the shortperiod comet group only by chance, their perihelion distance being lower than, but very close to, the $a_{\mathrm{J}}=5.2$ AU limit of the Jewitt (2009) definition.

Several of the bodies presently defined as active Centaurs also experienced close encounters with Saturn, which modified their orbit by decreasing their semi-major axis and perihelion distance, but still within the Jewitt's (2009) lower limit. Therefore, the historical distinctions among groups and families of active minor bodies are progressively being blended in the region of the solar system where these "transition" objects orbit for most of their lives. The study of their physical properties and in particular the characterisation of their cometary activity is a main topic in constraining the nature and the evolution of minor bodies in the solar system.

The active minor body $\mathrm{P} / 2010 \mathrm{C} 1$ (Scotti) (hereinafter P/2010 C1) was discovered on 9 February 2010 by J. V. Scotti with the Spacewatch $0.9-\mathrm{m}$ reflector at Kitt Peak. It showed a nearly stellar nuclear condensation with a long tail at PA $=283^{\circ}$ and was provisionally identified as a short-period comet. The long-term dynamical behaviour of $\mathrm{P} / 2010 \mathrm{C} 1$, as derived with the JPL Horizon integrator (Giorgini et al. 1996), is shown in Fig. 1: it suffered a close encounter with planet Saturn in 1877, which drastically decreased its perihelion distance and semimajor axis close to the present values of $q=5.235 \mathrm{AU}$ and $a=$ 7.065 AU, respectively.

Observations presented in this paper have been collected in the framework of a long term program aimed at searching for comet-like activity in several targets in the region outside Jupiter's orbit, to clarify which is the real fraction of active Centaurs in the group. In this paper, we present a portrait of $\mathrm{P} / 2010 \mathrm{C} 1$, whose comet-like activity has been characterised for the first time ever by means of multi-colour photometry.

The observations and data reduction are presented in Sect. 2. Section 3 summarises the observational results, while in Sect. 4 some considerations for the Centaur nucleus are derived. Summary and conclusions are given in Sect. 5.
Table 2. Geometry of observation of P/2010 C1 (Scotti).

\begin{tabular}{ccccc}
\hline \hline Date $^{a}$ & $r^{b}[\mathrm{AU}]$ & $\Delta^{c}[\mathrm{AU}]$ & $\mathrm{PA}^{d}[\mathrm{deg}]$ & $\alpha^{e}[\mathrm{deg}]$ \\
\hline 2 March 2011 03:33 UT & 5.508 & 4.643 & 280.2 & 5.32 \\
\hline
\end{tabular}

Notes. ${ }^{(a)}$ Beginning of image sequence. ${ }^{(b)}$ Heliocentric distance. (c) Geocentric distance. ${ }^{(d)}$ Position angle of the extended Sun-target radius vector, as seen in the observer's plane-of-sky, measured CCW. (e) Phase angle.

\section{Observations and data reduction}

The minor body P/2010C1 was observed on 2 March 2011 with the $3.52 \mathrm{~m}$ Telescopio Nazionale Galileo (TNG) at the Observatorio del Roque de los Muchachos (La Palma, Canary Islands). TNG images were obtained with the Device Optimised for the LOw RESolution (DOLORES) intrument, a focal reducer instrument installed at the Nasmyth B focus of the TNG. The detector is a $2048 \times 2048$ E2V 4240 thinned back-illuminated, deep-depleted, Astro-BB coated CCD with a pixel size of $13.5 \mu \mathrm{m}$. The scale is $0.252 \mathrm{arcsec} / \mathrm{px}$, which yields a field of view of about $8.6 \times 8.6 \mathrm{arcmin}$. The instrument was equipped with the broadband filters $B, V, R$, and $I$ of the Johnson-Cousins system. The circumstances of observations are summarised in Table 2 .

With the telescope tracking at the nonsidereal motion rate of $\mathrm{P} / 2010 \mathrm{C} 1,31$ consecutive images have been acquired (exposure time of $300 \mathrm{~s}$ each), with the sequence $R B V I R$ repeated many times, for a total of $9 R, 7 B, 7 \mathrm{~V}$, and $6 I$ exposures. The seeing was quite stable during the observations, with a mean value of $1.0^{\prime \prime}$ (FWHM) in the $R$ filter.

Despite the quite large heliocentric distance, $\mathrm{P} / 2010 \mathrm{C} 1$ was easily identifiable, and it appeared active in each single image for each filter. It appeared as an object with a central diffuse condensation and an extended sharp tail-like structure, oriented at PA = $284.5^{\circ}\left(14.5^{\circ} \mathrm{N}\right.$ of the $\left.\mathrm{W}\right)$, i.e. close the antisolar direction.

The images were reduced and calibrated using standard procedures (subtraction of master bias and flat-field correction), with the aid of the ESO's Munich Image Data Analysis System (MIDAS 1998) software package. The absolute calibration of the magnitudes was obtained computing the photometric zero points and extinction coefficients by a least square fit for several Landolt (1992) standard fields.

In each filter, the individual pre-reduced frames were quite homogeneous, both in the background values and in the general aspect of the Centaur coma and tail. To increase the signal-tonoise ratio for the image analysis, we decided to co-add the images obtained for each filter, grouped for similar airmass. Table 3 summarises the circumstances of image grouping and stacking.

The position of $\mathrm{P} / 2010 \mathrm{C} 1$ optocentre in each single image selected for the coadding was determined by fitting a twodimensional Gaussian to the innermost pixels of the coma. The images were then recentred using this optocentre and summed. Sky correction was performed by subtracting a first-order polynomial sky approximation computed from the pixel areas containing no stars. (The sky image was built for each frame via a standard MIDAS routine, with the areas for background chosen interactively via the cursor. In it, the sky image is created from a 2D polynomial, using the least-square method.) We obtained two final images for filters $B, V$ and $I$, and three final images for filter $R$. One example for these latter images is shown in Fig. 2. 
Table 3. Circumstances of image grouping and stacking.

\begin{tabular}{|c|c|c|c|c|c|c|}
\hline Filter & \# of images used for the analysis & \# of final stacks & stack id. & Time range $^{a}$ & Airmass sub-range & Median airmass $^{b}$ \\
\hline \multirow[t]{2}{*}{$B$} & 7 & 2 & $a(4 x)$ & $03: 41-04: 55$ & $1.116-1.201$ & 1.145 \\
\hline & & & $b(3 x)$ & $05: 32-06: 17$ & $1.298-1.504$ & 1.386 \\
\hline \multirow[t]{2}{*}{$V$} & 7 & 2 & $\mathrm{a}(4 \mathrm{x})$ & $03: 47-05: 01$ & $1.118-1.213$ & 1.151 \\
\hline & & & $b(3 x)$ & $05: 37-06: 23$ & $1.317-1.538$ & 1.412 \\
\hline \multirow[t]{3}{*}{$R$} & 9 & 3 & $\mathrm{a}(4 \mathrm{x})$ & $03: 33-04: 27$ & $1.114-1.153$ & 1.135 \\
\hline & & & $b(3 x)$ & $04: 50-05: 26$ & $1.190-1.280$ & 1.242 \\
\hline & & & $c(2 x)$ & $05: 49-06: 12$ & $1.362-1.471$ & 1.417 \\
\hline \multirow[t]{2}{*}{$I$} & 7 & 2 & $a(4 x)$ & $04: 44-05: 07$ & $1.121-1.227$ & 1.159 \\
\hline & & & $\mathrm{b}(2 \mathrm{x})$ & $05: 43-06: 06$ & $1.339-1.440$ & 1.390 \\
\hline
\end{tabular}

Notes. ${ }^{(a)}$ Night of 2011-03-02. ${ }^{(b)}$ Used for the photometric analysis of the stacked image.

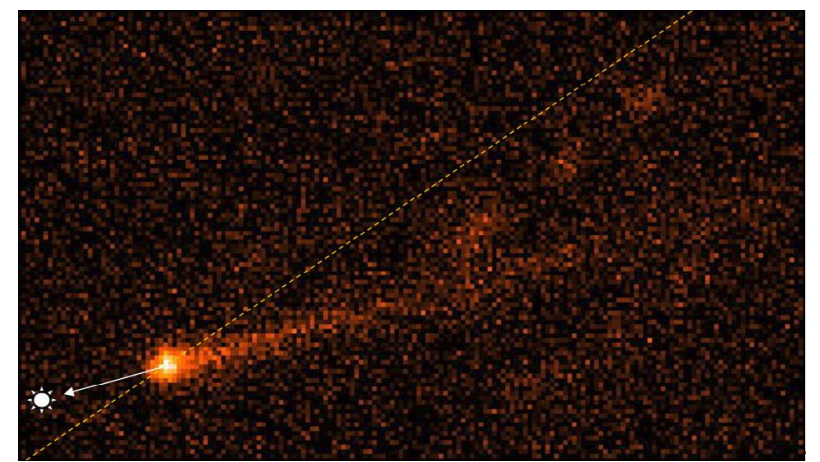

Fig. 2. One example of the final images of $\mathrm{P} / 2010 \mathrm{C} 1$ (Scotti) in the $R$ filter, obtained after co-adding of single frames taken at similar airmass (co-adding of 4 individual frames, for a total exposure time of $1200 \mathrm{~s}$. Median airmass 1.135). The linear scale is $1.5 \times 10^{5} \mathrm{~km} \times$ $8.5 \times 10^{4} \mathrm{~km}$. The dashed line is the projected comet's trajectory. The comet-Sun vector is also indicated. N is up, $\mathrm{E}$ is to the left.

\section{Observational results}

Each final image was individually analysed, and results were very compatible within measurement errors. Therefore, final values have been averaged to get the observational results presented in this section.

\subsection{Comet-like activity}

The TNG R band-pass filter (centred at $\lambda=625 \mathrm{~nm}$, with FWHM of $\sim 130 \mathrm{~nm}$ ) is the least affected by possibly present gaseous emission (even if it is unlikely that the gaseous emission in the visible range is dominant at $r=5.5 \mathrm{AU}$ ), and is therefore the most indicated for a detailed analysis of the central condensation, in order to verify that it is actually a dust coma. We summed up three co-added $\mathrm{R}$ images (with the same method as described in the previous section for each final image), and from this "master image" four profiles centred in the Centaur optocenter have been extracted and averaged to produce a mean target profile, as shown in Fig. 3. After normalisation to a value of 1 in the peak (the normalisation to 0 in the wings is ensured by the background subtraction), the profile has been compared with the stellar point spread function (PSF) profile, as extracted from several standard field stars in frames obtained just before and after the target sequence (average PSF has a FWHM of 1.1 arcsec). Similar methods have been successfully tested to enhance even weak dust coma in apparently stellar objects, mainly bare cometary nuclei (O'Ceallaigh et al. 1995; Licandro et al. 2000; Lowry \& Fitzsimmons 2005; Mazzotta Epifani et al. 2008).
The comparison is also shown in Fig. 3. There is clearly evident excess in the profile wings, not only in the quadrants where the tail-like structure extends, but also in the NE and SE quadrants (see also the residual points up to an optocentric distance of $1.3 \mathrm{arcsec}$ from the optocentre). This allows us to confirm the presence of a diffuse dust (sunlight reflecting) coma around the target nucleus, together with the sharp tail-like structure extending in the NW quadrant. We performed additional tests to secure the evidence of cometary activity in $\mathrm{P} / 2010 \mathrm{C} 1$ : similar results were obtained by comparing both (i) the stellar PSF profile (averaged before and after target imaging sequence) with target profile in each single image; and (ii) the "master image" profile with stellar profile from stars present in the same "master image", extracted perpendicularly to the apparent motion of the Centaur.

\subsection{Nucleus size}

P/2010 C1 appears to be exactly like a "typical" comet: a central nuclear condensation, surrounded by a diffuse coma and with a tail-like structure. To extract information on characteristics of the different part of the target, we performed aperture photometry of it (using the proper task defined in the MIDAS framework). We extracted photometric data from each stacked image (in each filter) and then performed a weighted average among the values obtained for each stack (see Table 3).

An accurate analysis of (standard) star aperture photometry showed that, for a point source, an aperture with a diameter of tewlve pixels from the optocentre includes the $90 \%$ of the total flux, a diameter of fourteen pixels includes the $93 \%$, and a diameter of sixteen pixels includes the $95 \%$. Less than the $10 \%$ of a point source is included in an annulus between a radius of six and eight pixels, so we decided to extract photometry for three apertures (centred on the optocentre) and listed in Table 4. The central region, $\mathfrak{R}_{3}$, is selected to represent the flux from the point nucleus, plus an unknown (even if presumably small) fraction of the coma contribute. The region $\mathfrak{R}_{3,6}$ is considered to contain contributions from both the nucleus and the coma. The region $\mathfrak{R}_{6,8}$ is assumed to be dominated by the coma.

The $\mathrm{R}$ magnitude in region $\mathfrak{R}_{3}$ can be used to estimate the upper limit to the geometric cross-section of the Centaur nucleus, using the expression of Russell (1916), derived for asteroids observed at large phase angle and conveniently reformulated by Jewitt (1991), which, in the case of a spherical object, is given by

$A a_{\mathrm{N}}^{2} \leq 2.24 \times 10^{22} r^{2} \Delta^{2} 10^{0.4\left(m_{\odot}-R+\beta \alpha\right)}$

where $A$ is the geometric albedo, $a_{\mathrm{N}}[\mathrm{m}]$ the radius of the target, $m_{\odot}$ the magnitude of the Sun in the same wavelength band of observations $\left(m_{\odot}=-27.10\right.$, see Holmberg et al. 2006), and $\alpha$ and $\beta$ 

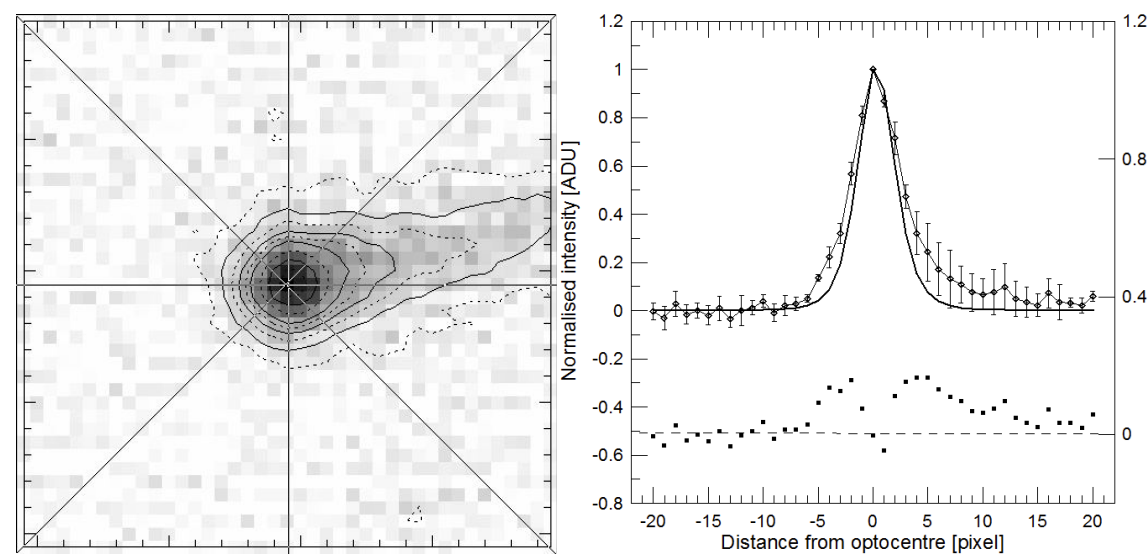

Fig. 3. Left: extraction of profiles, superimposed on the "master image" obtained for the $R$ filter (innermost $40 \times 40$ pixels; $\mathrm{N}$ is up, $\mathrm{E}$ is left), together with contour plots; right: comparison of the averaged profile (thin continuous line; error bars on points are due to dispersion of values) with the average stellar PSF profile (thick continuous line), together with the residual points (subtraction of the stellar profile to the target profile; black filled dots).

Table 4. Photometry regions of P/2010 C1 (Scotti).

\begin{tabular}{lcccccc}
\hline \hline $\begin{array}{l}\text { Region } \\
\text { label }\end{array}$ & $\begin{array}{c}\text { Region } \\
\text { shape }\end{array}$ & $\begin{array}{c}\text { Aperture radius } \\
\text { [pixel] }\end{array}$ & $\begin{array}{c}\text { Aperture radius, } \rho \\
{[\operatorname{arcsec}]}\end{array}$ & $\begin{array}{c}\text { Aperture radius, } r \\
{[\mathrm{~km}]}\end{array}$ & Mag & Dominant source \\
\hline $\mathfrak{R}_{3}$ & Circle & 3 & 0.76 & 2535 & $m_{3}$ & Nucleus [+ coma] \\
$\mathfrak{R}_{3,6}$ & Annulus & $3-6$ & $0.76-1.51$ & $2535-5070$ & $m_{3,6}$ & Nucleus + coma \\
$\mathfrak{R}_{6,8}$ & Annulus & $6-8$ & $1.51-2.02$ & $5070-6760$ & $m_{6,8}$ & Coma \\
\hline
\end{tabular}

Table 5. Photometry of P/2010 C1 (Scotti).

\begin{tabular}{lcccc}
\hline \hline Region & $B$ & $V$ & $R$ & $I$ \\
\hline$m_{3}$ & $23.79 \pm 0.05$ & $22.86 \pm 0.03$ & $22.37 \pm 0.02$ & $21.72 \pm 0.04$ \\
$m_{3,6}$ & $23.51 \pm 0.09$ & $22.81 \pm 0.06$ & $22.36 \pm 0.04$ & $21.48 \pm 0.08$ \\
$m_{6,8}$ & $24.33 \pm 0.09$ & $23.83 \pm 0.06$ & $23.34 \pm 0.05$ & $21.96 \pm 0.09$ \\
\hline
\end{tabular}

Notes. The results are the weighted mean among the 2 or 3 images (depending on filter) obtained for the image subsets (see text for details).

are the phase angle [deg] and the phase coefficient [mag/deg], respectively, in the phase-darkening function $\Phi(\alpha)=10^{-0.4 \alpha \beta}$. We assumed $m_{3}=22.37 \pm 0.02$ (see Table 5) as the nucleus magnitude (plus a small contribution from the near-nucleus coma).

As for the majority of minor bodies (with and without cometary activity) orbiting in the solar system, for $\mathrm{P} / 2010 \mathrm{C} 1$ we do not have values on the geometric albedo $A$ and the phase coefficient $\beta$. For the geometric albedo, the classical value of $A=0.04$ adopted for Jupiter-family comets (see Table 4 in Lamy et al. 2005 for range of measured values) is similar to the few measured values for other active objects like longperiod comets (Lamy et al. 2005; Abell et al. 2003; Jorda et al. 2000), but significantly lower than the albedo measured for other active Centaurs: 0.13 for Chiron (Bus et al. 1989) and 29P/Schwassmann-Wachmann 1 (Cruikshank \& Brown 1983). In its survey of Centaurs, Jewitt (2009) assumed a geometric albedo $A=0.1$ to compare size (and upper limits) among target objects. For the phase coefficient $\beta$, the commonly assumed value for the Jupiter family comets is $\beta=0.035 \mathrm{mag} / \mathrm{deg}$ (Snodgrass et al. 2011), while Peixinho et al. (2004) obtained a value of $\beta=0.11 \mathrm{mag} / \mathrm{deg}$ from a least-square fit of the linear $\Phi(\alpha)$ to the published data for the Centaur group. In its survey on Centaurs, Jewitt (2009) used a phase darkening function with a

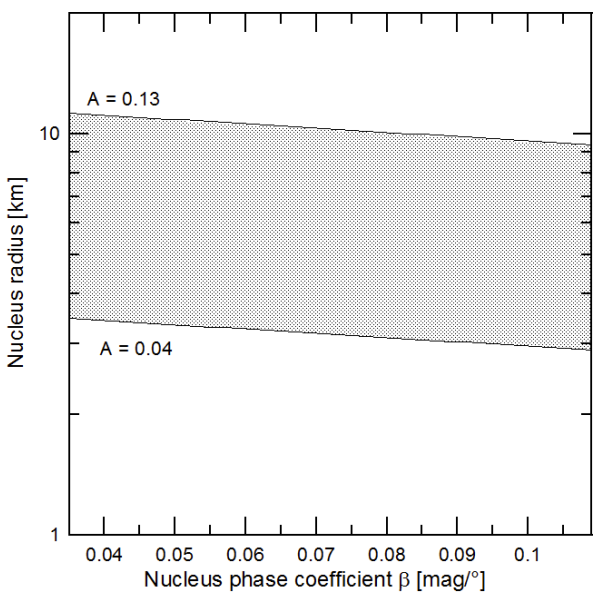

Fig. 4. P/2010 C1 (Scotti) nucleus size. The grey area indicates where the actual value for $\mathrm{P} / 2010 \mathrm{C} 1$ 's nucleus size probably lies, bracketting values for the two extreme values of geometric albedo $(A=0.04$ for Jupiter-family comets and $A=0.13$ for active Centaurs) and for a phase coefficient ranging among the extreme values of $\beta=0.035 \mathrm{mag} /{ }^{\circ}$ (Jupiter-family comets) and $0.11 \mathrm{mag} /{ }^{\circ}$ (Centaurs).

similar $\beta=0.1 \mathrm{mag} / \mathrm{deg}$. Figure 4 shows the region where probably the actual value of $\mathrm{P} / 2010 \mathrm{C} 1$ 's nucleus size lies.

To obtain a realistic assumption on upper limits for the nucleus size of $\mathrm{P} / 2010 \mathrm{C} 1$, we considered all the possible combination of the above values, and assumed the median value as a realistic estimate for this quantity. We obtained $a_{\mathrm{N}} \leq 4.8 \mathrm{~km}$. The latter is a very low value, which is more consistent with (even if slightly higher than) the nuclei of better observed Jupiter family comets $\left(a_{\mathrm{N}}=1.6 \mathrm{~km}\right.$, Lamy et al. 2005$)$ rather than typical values of tens of kilometres obtained for the upper limits of active Centaurs (Bauer et al. 2003; Jewitt 2009), with the exceptions of 39P/Oterma $\left(a_{\mathrm{N}} \leq 3 \mathrm{~km}\right.$, Jewitt 2009) and P/2004 A1 
Table 6. Colour indexes of $\mathrm{P} / 2010 \mathrm{C} 1$ (Scotti) in the photometry regions.

\begin{tabular}{cccccc}
\hline \hline & $\mathfrak{R}_{3}$ & $\mathfrak{R}_{3,6}$ & $\mathfrak{R}_{6,8}$ & Solar $^{a}$ & $\mathrm{JFC}^{b}$ \\
\hline$B-V$ & $0.92 \pm 0.05$ & $0.70 \pm 0.11$ & $0.50 \pm 0.11$ & 0.642 & 0.82 \\
$V-R$ & $0.49 \pm 0.03$ & $0.45 \pm 0.07$ & $0.49 \pm 0.08$ & 0.354 & 0.47 \\
$R-I$ & $0.65 \pm 0.05$ & $0.88 \pm 0.09$ & $1.38 \pm 0.10$ & 0.332 & 0.46 \\
$B-I$ & $2.06 \pm 0.06$ & $2.04 \pm 0.12$ & $2.37 \pm 0.13$ & 1.330 & 1.65 \\
\hline
\end{tabular}

Notes. ${ }^{(a)}$ Solar colours in the modern Johnson-Cousins system used by Landolt (1992) (from Holmberg et al. 2006). ${ }^{(b)}$ Average value, derived from the literature (Lamy et al. 2005, 2009; Snodgrass et al. 2005).

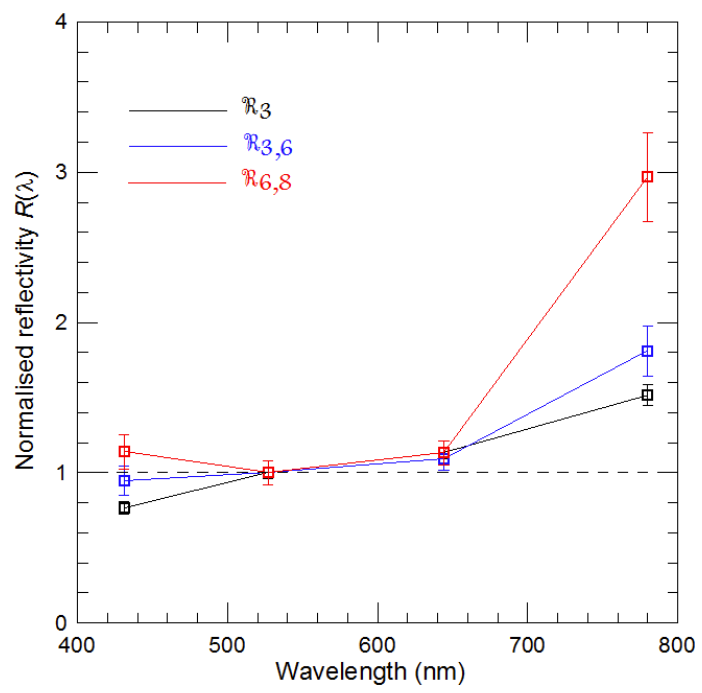

Fig. 5. Normalised reflectivity spectrum of the photometry regions of $\mathrm{P} / 2010 \mathrm{C} 1$ (Scotti). The spectra have been normalised to 1 at $\lambda=527 \mathrm{~nm}$ (the centre of $V$ filter). The horizontal dashed line represents a perfectly solar reflectivity.

(LONEOS) $\left(0.55 \leq a_{\mathrm{N}} \leq 3.5 \mathrm{~km}\right.$, Mazzotta Epifani et al. 2011). On the other hand, the radius of the observed inactive Centaurs is in general more than one order of magnitude larger (Bauer et al. 2003; Stansberry et al. 2008; Jewitt 2009; Perna et al. 2010).

\subsection{Nucleus and coma colours}

Table 6 summarises the colour indexes of $\mathrm{P} / 2010 \mathrm{C} 1$ obtained in each photometry region defined for the photometric analysis (see previous section). The information contained in the colour indexes can also be converted to a very low-resolution reflectivity spectrum for each region of the coma (Jewitt \& Meech 1986) using the following expression:

$\mathcal{S}(\lambda)=10^{-0.4\left(m(\lambda)-m_{\odot}(\lambda)\right)}$

where $m$ and $m_{\odot}$ are the magnitudes of the comet and of the Sun ${ }^{2}$. By normalising the reflectivity to 1 at the $V$ central wavelength, it becomes

$\mathcal{S}^{\prime}(\lambda)=10^{-0.4\left((m(\lambda)-m(V))-(m(\lambda)-m(V))_{\odot}\right)}$.

Figure 5 displays the normalised reflectivity of the three photometry regions of $\mathrm{P} / 2010 \mathrm{C} 1$.

From results summarised in Table 6 and Fig. 5, it is possible to conclude that the nucleus and the coma of $\mathrm{P} / 2010 \mathrm{C} 1$

\footnotetext{
${ }^{2} B_{\odot}=-26.098 ; V_{\odot}=-26.74 ; R_{\odot}=-27.094 ; I_{\odot}=-27.428 ;$ from Holmberg et al. (2006).
}

Table 7. Integrated magnitude and $\mathrm{A} f \rho$ of $\mathrm{P} / 2010 \mathrm{C} 1$ (Scotti).

\begin{tabular}{lcc}
\hline \hline Filter & Integrated magnitude & $\mathrm{A} f \rho[\mathrm{cm}]$ \\
\hline$B$ & $22.63 \pm 0.05$ & $28 \pm 2$ \\
$V$ & $21.88 \pm 0.03$ & $31 \pm 2$ \\
$R$ & $21.41 \pm 0.02$ & $34 \pm 2$ \\
$I$ & $20.51 \pm 0.04$ & $58 \pm 2$ \\
\hline
\end{tabular}

Notes. The reference aperture is $\phi=2.0^{\prime \prime}$, corresponding to $\rho=6.8 \times$ $10^{3} \mathrm{~km}$ at the target's distance. The results are the weighted mean among the 2 or 3 images (depending on filter) obtained for the image subsets (see text for details).

have likely different colours, even if both appear, in general, redder than the Sun. The nucleus region $\mathfrak{R}_{3}$ has colours $V-R=$ $0.49 \pm 0.03$, which are typical of nuclei of Jupiter family comets, but it appears redder in the $R-I$ and especially $B-I$ indexes than their nuclei. This redder colour is much more evident when going farther from the nucleus, in the photometry region dominated by the coma, where the reflectivity (see Fig. 5) at longer wavelengths is much higher than for the nucleus region.

\subsection{Af $\rho$ and dust-mass production rate}

The A $f \rho$ value expressed in cm (A'Hearn et al. 1984), where $A$ is the average grain albedo, $f$ the filling factor in the aperture field of view, and $\rho$ the linear radius of the aperture at the target, is usually considered as a proxy for the dust production and used to compare results obtained for active minor bodies. As a general rule, it is assumed that high A $f \rho$ values indicate high dust activity. At any rate, it should be taken into account that A $f \rho$ is an integrated quantity, and as such it involves several observational and physical parameters, sometimes difficult to compare: the aperture size, if the Af $\rho$ is not constant with the cometocentric distance; the geometrical albedo of the grains, which depends in turn on the observing phase and wavelength; the grain size distribution.

Our data for $\mathrm{P} / 2010 \mathrm{C} 1$ show that outside the region probably affected by the seeing $\left(\phi>0.55^{\prime \prime}\right)$, the Af $\rho$ is constant within the error bars for all the filters but $B$, probably the most affected by possible gas emission contamination, so depicting a dust environment consistent with a scenario of steady-state emission from the nucleus. Values for the reference aperture of $\phi=2.0^{\prime \prime}$ (the external edge of $\mathfrak{R}_{6,8}$, corresponding to $\rho=6.8 \times 10^{3} \mathrm{~km}$ ) are shown in Table 7 . In any case, these values should be regarded only as upper limits, since the contribution of the nucleus to photometry cannot be considered as negligible. Keeping in mind all the caveats listed above, a comparison with measured $R$-A $f \rho$ at similar heliocentric distance for other active objects shows that $\mathrm{P} / 2010 \mathrm{C} 1$ has a dust production rate that is more similar to that of the Jupiter family comets (e.g., $R$-A $f \rho=13 \mathrm{~cm}$ for comet P/1998 U4 (Spahr) at $r_{\mathrm{h}}=6 \mathrm{AU}$; see Table 5 in Mazzotta Epifani et al. 2008) than the one measured for active Centaurs (e.g., $R$-A $f \rho=163 \mathrm{~cm}$ for Centaur P/2004 A1 (LONEOS) at $r_{\mathrm{h}}=$ 6.5 AU; see Fig. 3 in Mazzotta Epifani et al. 2011)

To obtain a first-order quantitative estimate of the dust massloss rate for the centaur P/2010 C1 (Scotti), we applied a "photometric model", which relates the optical photometry to the dust production rate via some realistic assumptions on the cometlike dust environment. The method is derived from the one used by Jewitt (2009) to compute the dust production rate of active Centaurs in the region between 5 and $12 \mathrm{AU}$, and was adapted and already successfully applied by our group to several active 
minor bodies such as long period comets (Mazzotta Epifani et al. 2010, 2014), short period comets (Mazzotta Epifani \& Palumbo (2011), and active Centaurs (Mazzotta Epifani et al. 2011).

The method is fully described in our papers listed above. Here we recall only that the method consists of relating the total cross-section $C_{\mathrm{d}}$ of the coma dust particles, derived from the coma photometry, to the dust mass $M_{\mathrm{d}}$ of the coma itself, through adopting a power-law dust size distribution. The dustloss rate $Q_{\mathrm{d}}$ is finally obtained by considering the time of residence of dust grains in the projected coma portion (annulus).

For the purpose of estimating the dust mass-loss rate for the centaur P/2010 C1 (Scotti), we assume (as detailed above) that the photometry in the region $m_{6,8}$ describes the Centaur coma only. Therefore, the total coma cross-section is derived, following the expression by Russell (1916), as follows: $p_{R} \Phi(\alpha) C_{\mathrm{d}}=$ $2.25 \times 10^{22} \pi r^{2} \Delta^{2} 10^{-0.4\left(m_{\mathrm{d}}-m_{\odot}\right)}(r$ and $\Delta$ : heliocentric and geocentric distances; $\alpha$ : phase angle; $m_{\odot}$ : magnitude of the Sun in the $R$ filter; $m_{\mathrm{d}}$ : apparent $R$ magnitude in the region; $p_{R}$ : geometric albedo).

Unfortunately, since we do not know the characteristics of the dust environment of $\mathrm{P} / 2010 \mathrm{C} 1$ exactly (in particular, grain size distribution, albedo, and velocity), several (realistic) assumptions have to be made in order to derive quantitative estimates of the Centaur dust mass-loss rate.

Geometric albedo $p_{R}$ : the actual albedo of dust grains is unknown: we adopted the value of $p_{R}=0.1$ (Kolokolova 2004). This value is higher than the typical value for Jupiter family comet nuclei and is very compatible with the highest value measured for an active Centaur (0.13 for Chiron, Bus 1989). It has been assumed for similar estimates of dust mass-loss rate for active Centaurs by Jewitt (2009) and by Mazzotta Epifani et al. (2011)

Phase darkening $\Phi(\alpha)$ : the shape of this function depends upon a variety of properties of dust particles, including the distribution in size, roughness, and albedo. We adopted the "Schleicher's method" (Schleicher et al. 1998; Schleicher 2007), which reckons on the shallower dust comae phase function with respect to that of the bare cometary nucleus. Since $\mathrm{P} / 2010 \mathrm{C} 1$ was observed at a small phase angle $\left(5.32^{\circ}\right)$, we used the tabulated value of Schleicher's curve normalised at $0^{\circ}$ (asteroid.lowell.edu/comet/dustphaseHM_table.txt) $\Phi(\alpha)=$ $0.8205 \mathrm{mag}$.

Power-law dust size distribution: we adopted the value of $q=-3.5$ for grains between $a_{-}=10 \mu \mathrm{m}$ and $a_{+}$ranging from 1 to $10 \mathrm{~cm}$ for compact grains of bulk density $\rho=1000 \mathrm{~kg} / \mathrm{m}^{3}$. This is a realistic assumption supported also by numerical modelling of dust tails for some LPCs (Fulle et al. 1998; Korsun \& Chorny 2003; Mazzotta Epifani et al. 2009; Korsun et al. 2010) and JFCs (see a summary in Fulle 1999), and compatible with values adopted for similar estimates of dust mass-loss rate for active Centaurs (Jewitt 2009; Mazzotta Epifani et al. 2011). This results in an average grain scatterer of size $\langle a\rangle$ from $\sim 30$ to $100 \mu \mathrm{m}$, consistent with the observation that the dust coma of $\mathrm{P} / 2010 \mathrm{C} 1$ is reddish compared to the Sun.

Dust outflow velocity $v(r)$ : in the past, the application of an inverse numerical model to coma images allowed reconstruction of the dynamical evolution of the dust environment for a number of active targets, including the dust grain velocity (strictly dependent on dust size), at large heliocentric distance (e.g., Mazzotta Epifani et al. 2009; Korsun \& Chorny 2003). In this work, we considered a dust grain velocity between 1 and an estimated realistic upper limit of $50 \mathrm{~m} / \mathrm{s}$.

Table 8 summarises the values for the model quantities as described above, while Table 9 summarises the model output for
Table 8. Summary of parameters adopted in the photometric model applied to $\mathrm{P} / 2010 \mathrm{C} 1$ (Scotti).

\begin{tabular}{lc}
\hline \hline Parameter & Value \\
\hline Albedo & 0.1 \\
Phase function & $0.8205 \mathrm{mag}$ \\
Dust distrib. power index & -3.5 \\
Grain size range: & lower limit: $10 \mu \mathrm{m}$ \\
& upper limit: $1-10 \mathrm{~cm}$ \\
Grain velocity range & $1-50 \mathrm{~m} \mathrm{~s}^{-1}$ \\
\hline
\end{tabular}

Table 9. Model dust mass-loss rate for P/2010 C1 (Scotti).

\begin{tabular}{lcccc}
\hline \hline$C_{\mathrm{d}}^{a}\left[\mathrm{~m}^{2}\right]$ & \multicolumn{2}{c}{$M_{\mathrm{d}}^{b}[\mathrm{~kg}]$} & \multicolumn{2}{c}{$Q_{\mathrm{d}}^{c}[\mathrm{~kg} / \mathrm{s}]$} \\
& Lower limit & Upper limit & Lower limit & Upper limit \\
\hline $3.79 \times 10^{6}$ & $1.6 \times 10^{5}$ & $5.1 \times 10^{5}$ & 0.1 & 15 \\
\hline
\end{tabular}

Notes. ${ }^{(a)}$ Derived dust cross-section. ${ }^{(b)}$ Derived dust mass in the coma annulus. The lower limit corresponds to grains in the range $10 \mu \mathrm{m}-1 \mathrm{~cm}$, The upper limit corresponds to grains in the range $10 \mu \mathrm{m}-10 \mathrm{~cm} .{ }^{(c)}$ Derived dust mass-loss rate: the lower limit corresponds to grains in the range $10 \mu \mathrm{m}-1 \mathrm{~cm}$ crossing the projected coma annulus at $v=1 \mathrm{~m} / \mathrm{s}$, the upper limit corresponds to grains in the range $10 \mu \mathrm{m}-10 \mathrm{~cm}$ crossing the projected coma annulus at $v=50 \mathrm{~m} / \mathrm{s}$.

$\mathrm{P} / 2010 \mathrm{C} 1$. It appears clear, also in comparison with dust massloss rate values computed for several active minor bodies of different families and groups (see Mazzotta Epifani et al. 2014 and in particular their Fig. 6), that $\mathrm{P} / 2010 \mathrm{C} 1$ is a rather weak dust emitter, with a dust-loss rate comparable to that of the weakest SPCs at shorter heliocentric distances and to the weakest active Centaur 39P/Oterma (Jewitt 2009).

\section{Summary and conclusions}

We performed multicolour broad-band photometry of the active Centaur P/2010 C1 (Scotti), observed at the heliocentric distance $r=5.5$ AU with the $3.52 \mathrm{~m}$ TNG telescope. Our main results can be summarised as follows.

1. $\mathrm{P} / 2010 \mathrm{C} 1$ has a $R-\mathrm{A} f \rho=34 \pm 2 \mathrm{~cm}$. This value is more similar to the few measured for Jupiter-family comets at similar heliocentric distances than those of the few other active Centaurs.

2. The nucleus of $\mathrm{P} / 2010 \mathrm{C} 1$ has visual colour $V-R=0.49 \pm$ 0.03 , which is typical of the nuclei of Jupiter-family comets, but instead appears redder than cometary nuclei in the colour indexes $R-I=0.65 \pm 0.05$ and $B-I=2.06 \pm 0.06$. In the photometric region dominated by the dust coma, the reflectivity at longer wavelengths is even higher $(B-I=2.37 \pm 0.13)$.

3. We derived an upper limit from photometry for the Centaur nucleus of $a_{\mathrm{N}} \leq 4.8 \mathrm{~km}$. This result seems to confirm that the size of $\mathrm{P} / 2010 \mathrm{C} 1$ is quite small, which is more consistent with (even if higher than) the nuclei of Jupiter family comets, and is similar to the value derived for the smallest active Centaurs (39P/Oterma and P/2004 A1 (LONEOS). Its nucleus size is very likely more than one order of magnitude smaller than the values obtained for the inactive Centaurs.

4. By means of the application of the "photometric model" and some realistic assumptions on the comet-like dust environment, we derived a dust mass-loss rate range of $0.1-15 \mathrm{~kg} / \mathrm{s}$. This realistic range depicts the Centaur as a rather weak dust emitter, with a dust-loss rate more comparable to that of the weakest Jupiter-family comets and active Centaurs. 
All the results obtained during the observation run and summarised above confirm that the active Centaur P/2010 C1 (Scotti) can be considered a good example of a "transition object" between the two groups of Centaurs and Jupiter-family comets.

The historical distinctions among groups and families of active minor bodies are therefore being continuously blended, especially in the region outside the Jupiter orbit, where they spend most of their life inside the solar system, once they are presumably injected inwards from the Kuiper Belt. The complete characterisation of the comet-like activity of objects outside Jupiter's orbit, like the one presented in this paper for P/2010 C1 (Scotti) and presently available only for few other bodies, poses important constraints to fully depict the scenario of the dynamical and physical evolution of minor bodies coming from the extremely far regions of our solar system, and slowly travelling towards the innermost part, close to the Earth and even beyond.

Acknowledgements. We gratefully acknowledge funding from Italian Space Agency (ASI) under contract I/062/08/00. J.R.B. aknowledges funding from MIUR-PRIN 2008. J.L. aknowledges support from the Spanish "Ministerio de Ciencia e Innovación" project AYA2011-29489-C03-02.

\section{References}

Abell, P. A., Fernandez, Y. R., Pravec, P., et al. 2003, in Lunar and Planetary Science XXXIV, Abstract \# 1253 (Houston: Lunar and Planetary Institute)

A'Hearn, M. F., Schleicher, D. G., Feldman, P. D., Millis, R. L., \& Thompson, D. T. 1984, AJ, 89,579

Bauer, J. M., Meech, K. J., Refandez, Y. R., et al. 2003, Icarus, 166, 195 Bauer, J. M., Gray, T., Blauvelt, E., et al. 2013, ApJ, 773, 22

Boattini, A., Hill, R. E., Birtwhistle, P., et al. 2008, IAU Circ., 8988, 1 Bressi, T. H., Stevens, B. L., Durig, D. T., et al. 2013, MPEC, 2013-D22 Bus, S. J., Bowell, E., Harris, A. W., \& Hewiit, J. V. 1989, Icarus, 77, 223 Buzzi, L., Marinelli, M., Di Michele, V., et al. 2007, MPEC, 2007-U65 Cartwright, I. M., Fitzsimmons, A., Williams, I. P., \& Kemp, S. N. 1997, Planet. Space Sci., 45, 7, 821

Capria, M. T., Coradini, A., De Sanctis, M. C., \& Orosei, R. 2000a, A\&A, 357, 359

Capria, M. T., Coradini, A., De Sanctis, M. C., \& Orosei, R. 2000b, AJ, 119, 3112

Choi, Y.-J., Weissman, P. R., \& Polishook, D. 2006, IAU Circ., 8656

Cruikshank, D. P., \& Brown, R. H. 1983, Icarus, 56, 377

Epifani, E., Rotundi, A., Foster, M. J., et al. 1999, Planet. Space Sci., 47, 765

Fernandez, Y. R., Meech, K. J., Pittichova, J., et al. 2001, IAU Circ., 7689

Fink, U., Fevig, R. A., Tegler, S. C., \& Romanishin, W. 1997, Planet. Space Sci., 45,1383

Fulle, M. 1999, Planet. Space Sci., 47, 827

Fulle, M., Cremonese, G., \& Bohm, C. 1998, AJ, 116, 1470

Gibbs, A. R., Sarneczky, K., Scotti, J. V., et al. 2011a, IAU Circ., 9199, 1

Gibbs, A. R., Tornero, S. F., \& Williams, G. V. 2011b, IAU Circ., 9234, 1

Giorgini, J. D., Yeomans, D. K., Chamberlin, A. B., et al. 1996, BAAS, 28, 3, 1158

Green, D. W. E. 1998, IAU Circ., 7050, 3

Green, D. W. E. 2004, IAU Circ., 8430, 3

Green, D. W. E. 2005, IAU Circ., 8618, 2

Green, D. W. E. 2010, IAU Circ., 9145, 2

Hahn, G., \& Lagerkvist, C.-I. 2010, Astron. Nachr., 331, 692

Holman, M. J., \& Wisdom, J. 1993, AJ, 105, 1987

Holmberg, J., Flynn, C., \& Portinari, L. 2006, MNRAS, 367, 449

Jewitt, D. C. 1990, ApJ, 351, 277

Jewitt, D. C. 1991, in Comets in the Post-Halley Era, 1, eds. R. L. Newburn, M. Jr. Neugebauer, J. Rahe (Kluwer Acad. Publ.)

Jewitt, D. 2009, AJ, 137, 4296

Jewitt, D. C., \& Meech, K. J. 1986, AJ, 310, 937

Jorda, L., Lamy, P., Groussin, O. et al. 2000, in Proc. ISO beyond Point Sources: Studies of the extended Infrared Emission, ed. R.L. Laureijs et al., Noordwijk, The Netherlands, ESA SP, 455

Kolokolova, L., Hanner, M. S., Levasseur-Regourd, A.-C., \& Gustafson, B. 2004, in Comets II, eds. M. Festou, H. U. Keller, \& H. A. Weaver (Tucson: Univ. Arizona Press)
Korsun, P. P., \& Chorny, G. F. 2003, A\&A, 410, 1029

Korsun, P. P., Kulyk, I. V., Ivanova, O. V., et al. 2010, Icarus, 210, 916

Kowal, C. T., Liller, W., \& Chaisson, L. J. 1977, IAU Circ., 3147

Kowal, C. T., Liller, W., \& Marsden, B. G. 1979, in Dynamics of the Solar System, ed. R. L. Duncombe (Dordrecht: Kluwer), IAU Symp., 81, 245

Kusnirak, P., \& Balam, D. 2000, IAU Circ., 7368

Lacerda P. 2013, MNRAS, 428, 1818

Lagerkvist, C.-I., Hahn, G., Karlsson, O., \& Carsenty, U. 2000, A\&A, 362, 406

Lamy, P., Toth, I., Fernandez, Y. R., \& Weaver, H. A. 2004, in Comets II, eds. M. Festou, H. U. Keller, \& H. A. Weaver (Tucson: Univ. Arizona Press)

Lamy, P. L., Toth, I., Weaver, H. A., A'Hearn, M. F., \& Jorda, L. 2009, A\&A, 508, 1045

Landolt, A. U. 1992, AJ, 104, 340

Lara, L.-M., Licandro, J., Oscoz, A., \& Motta, V. 2003, A\&A, 497, 843

Lawrence, K. J., Helin, E. F., Pravdo, S., Dyvig, R., \& Balam, D. D. 2001, IAU Circ., 7654, 1

Levison, H. F., \& Duncan, M. J. 1997, Icarus, 127, 13

Licandro, J., Tancredi, G., Lindgren, M., Rickman, H., \& Gil Hutton, R. 2000, Icarus, 147, 161

Lowry, S. C., \& Fitzsimmon, A. 2005, MNRAS, 358, 641

Lowry, S. C., Fitzsimmons, A., Cartwright, I. M., \& Williams, I. P. 1999, A\&A, 349,649

Luu, J. X., \& Jewitt, D. C. 1990, AJ, 100, 913

Mazzotta Epifani, E., \& Palumbo, P. 2011, A\&A, 525, A62

Mazzotta Epifani, E., Palumbo, P., Capria, M. T., et al. 2006, A\&A, 460, 935

Mazzotta Epifani, E., Palumbo, P., Capria, M. T., et al. 2008, MNRAS, 390, 265

Mazzotta Epifani, E., Palumbo, P., Capria, M. T., et al. 2009, A\&A, 502, 355

Mazzotta Epifani, E., Dall'Ora, M., Di Fabrizio, L., et al. 2010, A\&A, 513, A33

Mazzotta Epifani, E., Dall'Ora, M., Perna, D., Palumbo, P., \& Colangeli, L. 2011, MNRAS, 415, 3097

Mazzotta Epifani, E., Perna, D., Di Fabrizio, L., et al. 2014, A\&A, 561, A6

Meech, K. J., \& Belton, M. 1989, IAU Circ., 4770

Meech, K. J., \& Svoren, J. 2004, in Comets II, eds. M. Festou, H. U. Keller, \& H. A. Weaver (Tucson: Univ. Arizona Press)

Meech, K. J., Bauer, J. M., \& Hainaut, O. R. 1997, A\&A, 326, 1268

Meech, K. J., Pittichova, J., Bar-Nun, A., et al. 2009, Icarus, 201, 719

Melita, M. D., \& Licandro, J. 2012, A\&A, 539, A144

Meyer, M. 2003, IAU Circ., 8153, 3

Miller, P., Roche, P., Tripp, A., et al. 2012, MPEC 2012-R08

O'Ceallaigh, D. P., Fitzsimmons, A., \& Williams, I. P. 1995, A\&A, 297, L17

Notesco, G. A., Bar-Nun, A., \& Owen, T. 2003, Icarus, 162, 183

Peixinho, N., Doressoundiram, A., Delsanti, A., et al. 2003, A\&A, 410, L29

Peixinho, N., Boehnhardt, H., Belsakya, I., et al. 2004, Icarus, 170, 153

Peixinho, N., Delsanti, A., Guilbert-Lepoutre, A., Gafeira, R., \& Lacerda, P. 2012, A\&A, 546, A86

Perna, D., Barucci, M. A., Fornasier, S., et al. 2010, A\&A, 510, A53

Pravdo, S., Helin, E. F., Hicks, M., \& Lawrence, K. 2001, IAU Circ., 7738, 1

Prialnik, D. 1997, ApJ, 478, 547

Prialnik, D., Brosch, N., \& Ianovici, D. 1995, MNRAS, 276, 1148

Primak, N., Schultz, A., Watters, S., et al. 2011, IAU Circ., 9225, 1

Read, M. T., \& Scotti, J. V. 2005, IAU Circ., 8614, 2

Romanishin, W., \& Tegler, S. C. 2005, IAU Circ., 8545, 1

Romon Martin, J., Delahodde, C., Barucci, M. A., de Bergh, C., \& Peixinho, N. 2003, A\&A, 400, 369

Russell, H. N. 1916, ApJ, 43, 173

Schleicher, D. G. 2007, Icarus, 190, 406

Schleicher, D. G., Millis, R. L., \& Birch, P. V. 1998, Icarus, 132, 397

Schulz, R., Stuewe, J. A., Boehnhardt, H., Gaessler, W., \& Tozzi, G. P. 2003, A\&A, 398, 345

Schulz, R., Stuewe, J. A., \& Boehnhardt, H. 2004, in The NEW Rosetta Targets: Observations, Simulations and Instrument Performances, eds. L. Colangeli, E. Mazzotta Epifani, \& P. Palumbo (Dordrecht: Kluwer Acad. Pub.)

Scotti, J. V. 2010, IAU Circ., 9116, 1

Scotti, J. V., Bressi, T. H., Spahr, T. B., et al. 2009, MPEC 2009-F28

Scotti, J. V., Gilmore, A. C., Kilmartin, P. M., \& Marsden, B. G. 2010, MPEC 2010-N12

Skiff, B. A., Miller, H. R., \& Young, J. 2004, IAU Circ., 8267

Snodgrass, C., Fitzsimmons, A., \& Lowry S. C. 2005, A\&A, 444, 287

Snodgrass, C., Fitzsimmons, A., Lowry, S. C., \& Weissmann, P. 2011, MNRAS, 414,458

Stansberry, J., Grundy, W., Brown, M., et al. 2008, in The Solar System Beyond Neptune, eds. M. A. Barucci, H. Boehnhardt, D. P. Cruikshank, \& A. Morbidelli (Tucson: Univ. Arizona Press)

Tichy, M., Moravec, Z., Offutt, W., \& Pravec, P. 1997, IAU Circ., 6755, 1 